\title{
The Design of Temperature Control System for Rice Wine Fermentation
}

\author{
ZHANG Shengyi and WANG Xinming, \\ HuBei Engineering University of School of Physics and Electronic Information Engineering, Xiaogan, \\ Hubei, China \\ Zsyzsy6445@163.com
}

Keywords: Fermentation temperature; STM32; control system; temperature control module

\begin{abstract}
Fermentation temperature is the key to decide the quality of the rice wine, so the temperature controlling system in fermentation room is established base on stm32 microcontroller and digital temperature sensor. The whole block diagram for the system is firstly detailed, and then the Temperature collecting module and temperature control module are also described. At last the software flow chart is discussed. The experiment is done based on designed system, and the data is analyzed. The result show that designed system can realize high accuracy temperature controlling.
\end{abstract}

\section{Introductions}

Fermentation technology is one of the important processes in the rice wine production and Fermentation temperature is the key to decide the quality of the rice wine ${ }^{[1][2]}$. If the fermentation temperature is controlled within desired environment, the needed quantity for material could be reduced and the quality of the rice wine could be greatly improved. Face to the increment of the production, the conventional manual temperature controlling encounters a lot of difficulty ${ }^{[3][4]}{ }^{[5]}$. In contrast, the automatic control of by means of microcontroller, digital sensor and some implement part can be widely used in the process of fermentation.

According to above description, the temperature control system is designed and some experiment is done. In this paper, the temperature control system is firstly detailed, including whole block diagram for the system, the control core STM32F103, Temperature collecting module, the drive circuit for the temperature control module and software flow chart designs. Then, the experiment is done based on designed system. At last, the data is analyzed and some conclusion is derived.

\section{The design for temperature control system}

The whole block diagram for the system. The system block diagram for the control system with the control core of STM32F103 controller is shown in figure 1. It is consisted of temperature collecting module, temperature control module and MCU minimum system. The sensor for temperature collecting module is DS18B20, with the character of 1-Wire interface in which all of the data, address and command can be transmitted. Temperature control module is consisted of the heating part and cooling part, it is separately electric incandescent lamp and fans. The system except MCU minimum system is mounted inside the fermentation room.

In this system, the temperature of the fermentation room can be real-time collected by the sensor and sent to microcontroller, if the temperature is above setting value, the fans should be startup; otherwise, electric incandescent lamp is lightened. Further, the FLASH, SRAM and 
keyboard is expanded

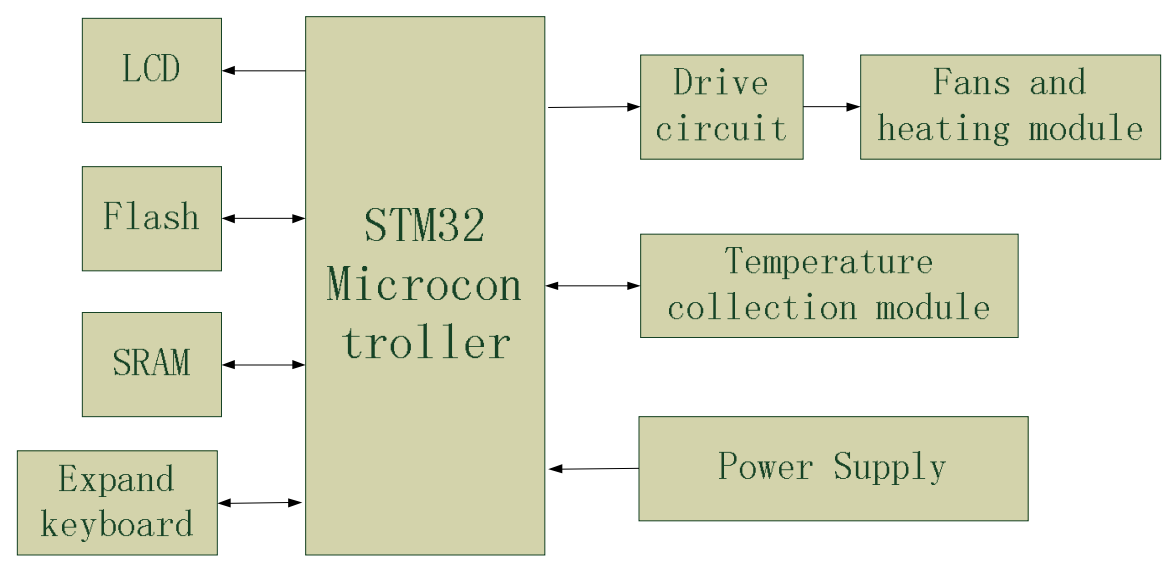

Fig. 1 the system block diagram for the control system

The Temperature collecting module. The temperature collecting sensor in this paper is the Digital Thermometer DS18B20 Which provides 9 to 12-bit temperature readings ${ }^{[6]}{ }^{[7]}$. Because of the Information sent to/from the DS18B20 over a 1-Wire interface, only one wire (and ground) needs to be connected from a central microprocessor to a DS18B20. Power for reading, writing, and performing temperature conversions can be derived from the data line itself with no need for an external power source.

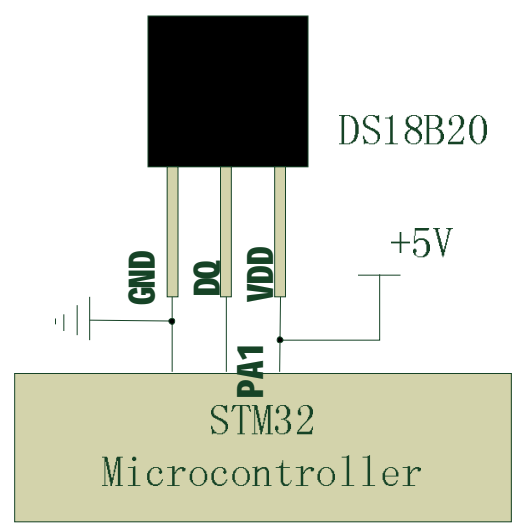

Fig.2 diagrammatic sketch Wire Connected between sensor and microcontroller

Because each DS18B20 contains a unique silicon serial number, multiple DS18B20s can exist on the same 1-Wire bus. This allows for placing temperature sensors in many different places. Applications where this feature is useful include HVAC environmental controls, sensing temperatures inside buildings, equipment or machinery, and process monitoring and control.

The drive circuit for the temperature control module. The drive circuit of temperature control module is illustrated in figure 3, the electric incandescent lamp and fans is drive by $220 \mathrm{AVC}$, while theirs control is finished through relay K1 and K2. Because it need large current for driving of relay $\mathrm{K} 1$ and K2, the ULN2003 with high voltage and high current darlington pairs is adopted. The control command of lamp and fans is separately input from channel of IN1 and IN2 of ULN2003, these signal is enlarged and drive the relay action. 


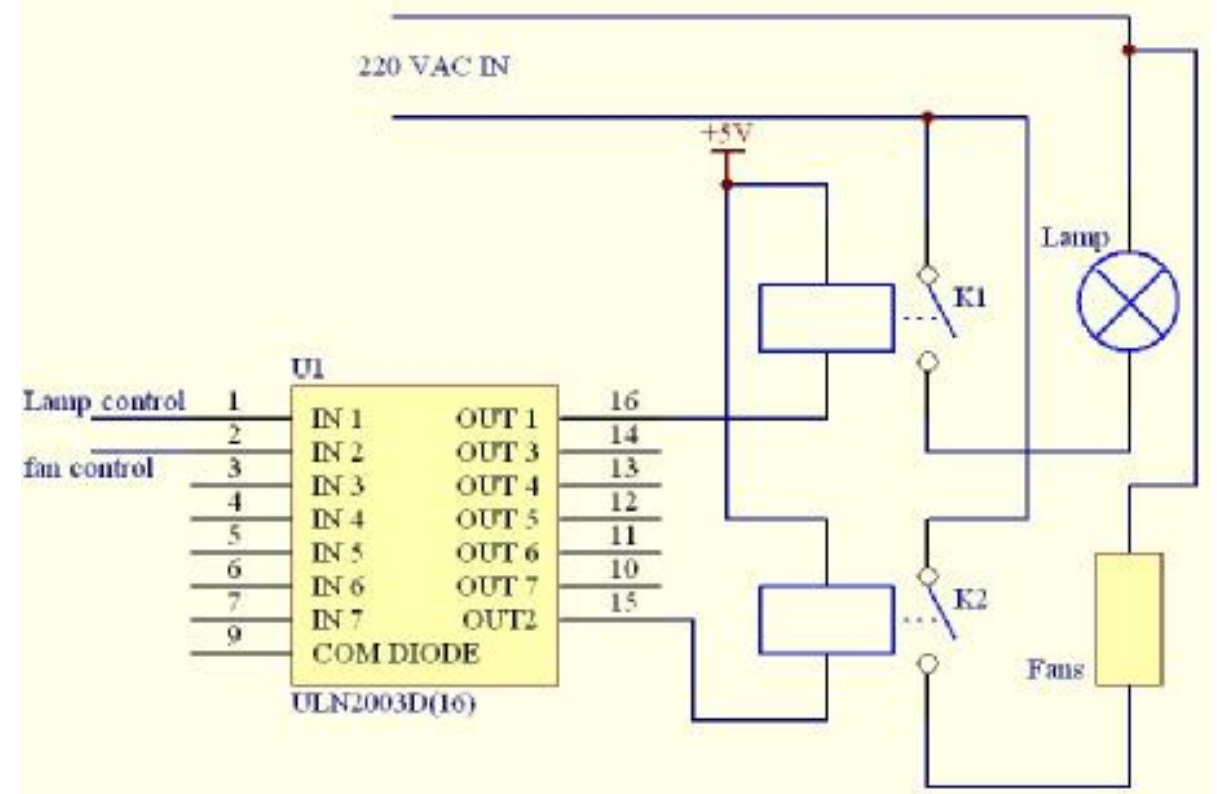

Fig.3 The circuit of temperature control module

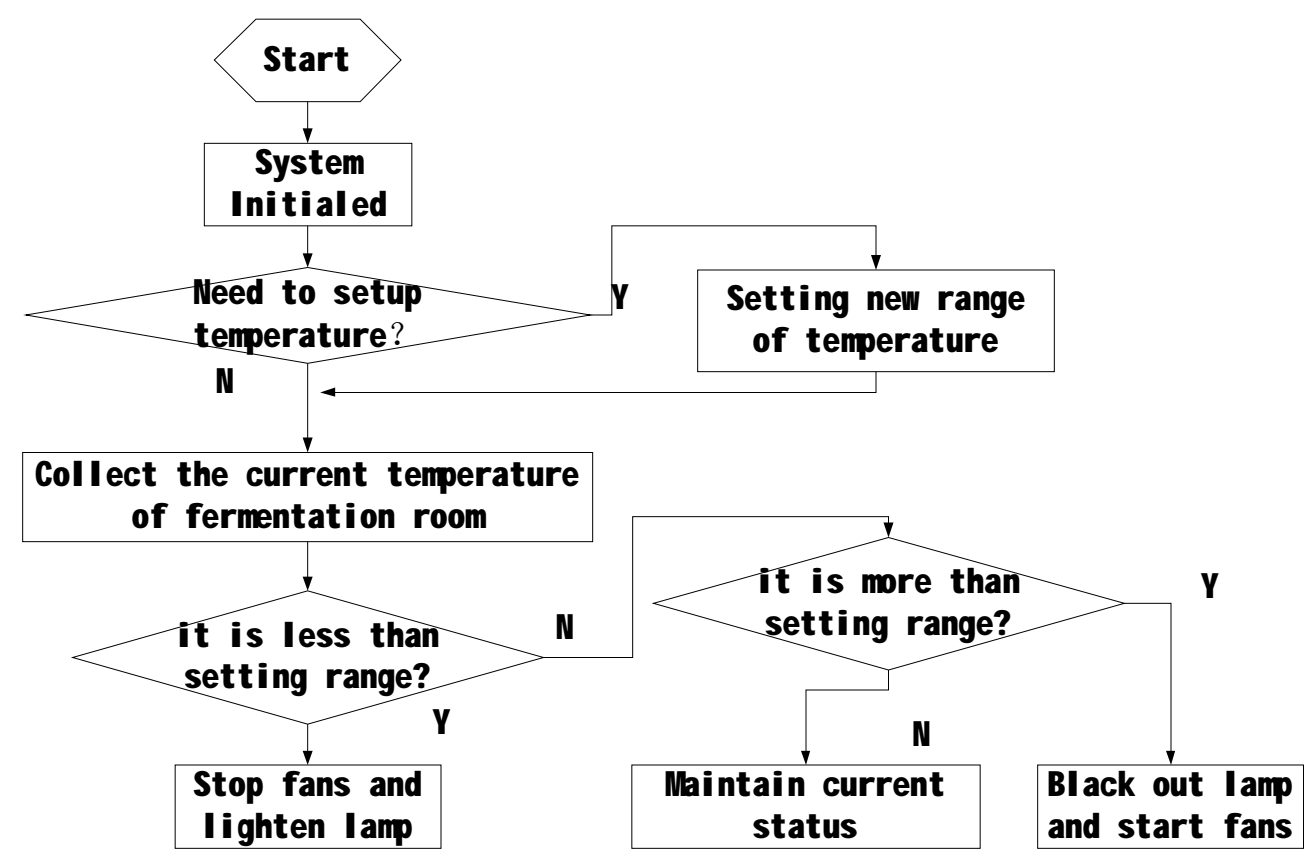

Fig. 4 the flow chart for the temperature control system of Fermentation room

Software designs for the designed system. The flow chart of the system software is illustrated in figure 4 . When the power supply is switched on, the system will enter into initial state. If the new temperature range of the fermentation room is set, the current temperature is collected and it enters into switch select. If the current value is less than setting range, the hearting module is started and the cooling module is stopped; if the current value is more than setting range, the hearting module is stopped and the cooling module is started; otherwise, this system should be maintained current status.

\section{Experiment and result}


At last, the temperature control experiment in fermentation room is done with the same initial indoor temperature of $20^{\circ} \mathrm{C}$. Because of the perfect fermentation temperature is $28-32^{\circ} \mathrm{C}$, so the five different setting temperature of $28^{\circ} \mathrm{C}, 29^{\circ} \mathrm{C}, 30^{\circ} \mathrm{C}, 31^{\circ} \mathrm{C}$ and $32^{\circ} \mathrm{C}$ is selected for experiment, both the temperature after adjusted and the time consumption for temperature control are recorded the experimental result for temperature control is shown in table 1.

Tab. 1 experimental result for temperature control

\begin{tabular}{cccc}
\hline Initial value $\left({ }^{\circ} \mathrm{C}\right)$ & preset value $\left({ }^{\circ} \mathrm{C}\right)$ & Adjusted value $\left({ }^{\circ} \mathrm{C}\right)$ & Adjust time(s) \\
\hline 20 & 28 & 28.4 & 101 \\
20 & 29 & 28.8 & 103 \\
20 & 30 & 29.8 & 104 \\
20 & 31 & 31.1 & 103 \\
20 & 32 & 31.8 & 102 \\
\hline
\end{tabular}

From the table, the temperature after adjusted is near is setting value, and within permissible error and the time consumption is almost same. So we can infer that the designed system can reach high accuracy temperature controlling and it reaches desired function.

\section{Conclusions}

To achieve high accuracy temperature controlling for rice wine fermentation room, the experiment is founded through temperature collecting module, temperature control module and MCU minimum system. Based on designed system, the experiment is done, and the result shows that designed system can realize high accuracy temperature controlling.

\section{References}

[1] Zhu Y, Zhang J, Shi Z, Mao Z. Optimization of operating conditions in rice heat blast process for Chinese rice wine production by combinational utilization of neural network and genetic algorithms. J Inst Brew, 2004,110:117-123

[2] Gohel V, Duan G. Conventional process for ethanol production from Indian broken rice and pearl millet. Bioprocess Biosyst Eng ,2012, 35:1297-1308

[3] Wang J, Zhang Y, Yu Q . Determination of alcohol and total acid content in Miaofu rice wine by near infrared spectroscopy. China Brewing ,2011,11:168-170

[4] Mo X, Xu Y, Fan W. Characterization of aroma compounds in Chinese rice wine Qu by solvent-assisted flavor evaporation and headspace solid-phase microextraction. J Agric Food Chem,2010, 58:2462-2469

[5] Jin Y, Fan W, Xu Y, Zhao G. Research on the tolerance of yellow rice wine yeast. Liquor-Mak Sci and Technol,2008, 6:17-21

[6] Gu ZY, Liu LY, Du ZH. DS18B20 C language programming interface. Microcontrollers Embeded Syst, 2002, 7:22-24

[7] Liu JT, Mao SK. Principle of DS18B20 and its interface design based on C. Instrum Meters User, 2005,12(6):138-140 